Research Paper

\title{
SCAPs Regulate Differentiation of DFSCs During Tooth Root Development in Swine
}

\author{
Xiaoshan $\mathrm{Wu}^{1}{ }^{1,2}$, Lei $\mathrm{Hu}^{2}$, Yan $\mathrm{Li}^{2}$, Yang $\mathrm{Li}^{2}$, Fu Wang 3 , Ping $\mathrm{Ma}^{2}$, Jinsong Wang2 , Chunmei Zhang2, Canhua \\ Jiang ${ }^{\circledR}$, Songlin Wang ${ }^{2 \bowtie}$ \\ 1. Department of Oral and Maxillofacial Surgery, Xiangya Hospital, Central South University; \\ 2. Molecular Laboratory for Gene Therapy and Tooth Regeneration, Beijing Key Laboratory of Tooth Regeneration and Function Reconstruction, Capital \\ Medical University School of Stomatology, Beijing, China; \\ 3. Department of Oral Basic Science, School of Stomatology, Dalian Medical University, Dalian, China \\ $\triangle$ Corresponding authors: Songlin Wang, Phone: 86-10-83950127; Email: slwang@ccmu.edu.cn; Canhua Jiang, Phone: 86-731-89753046; Email: \\ canhuaj@aliyun.com \\ (C) Ivyspring International Publisher. This is an open access article distributed under the terms of the Creative Commons Attribution (CC BY-NC) license \\ (https://creativecommons.org/licenses/by-nc/4.0/). See http://ivyspring.com/terms for full terms and conditions.
}

Received: 2017.08.23; Accepted: 2017.12.23; Published: 2018.01.19

\begin{abstract}
The tooth root transmits and balances occlusal forces through the periodontium to the alveolar bone. The periodontium, including the gingiva, the periodontal ligament, the cementum and the partial alveolar bone, derives from the dental follicle (DF), except for the gingiva. In the early developmental stages, the DF surrounds the tooth germ as a sphere and functions to promote tooth eruption. However, the morphological dynamics and factors regulating the differentiation of the DF during root elongation remain largely unknown. Miniature pigs are regarded as a useful experimental animal for modeling in craniofacial research because they are similar to humans with respect to dentition and mandible anatomy. In the present study, we used the third deciduous incisor of miniature pig as the model to investigate the factors influencing DF differentiation during root development. We found that the DF was shaped like a crescent and was located between the root apical and the alveolar bone. The expression levels of WNT5a, $\beta$-Catenin, and COL-I gradually increased from the center of the DF (beneath the apical foramen) to the lateral coronal corner, where the DF differentiates into the periodontium. To determine the potential regulatory role of the apical papilla on DF cell differentiation, we co-cultured dental follicle stem cells (DFSCs) with stem cells of the apical papilla (SCAPs). The osteogenesis and fibrogenesis abilities of DFSCs were inhibited when being co-cultured with SCAPs, suggesting that the fate of the DF can be regulated by signals from the apical papilla. The apical papilla may sustain the undifferentiated status of DFSCs before root development finishes. These data yield insight into the interaction between the root apex and surrounding DF tissues in root and periodontium development and shed light on the future study of root regeneration in large mammals.
\end{abstract}

Key words: tooth root; root apex; dental sac; regeneration; mammals; Sus scrofa

\section{Introduction}

The dental follicle (DF) originates from cranial neural crest cells and is a loose connective tissue sac that plays critical roles in multiple stages of tooth development [1-3]. The DF is formed at the cap stage and surrounds the developing tooth germ as a sphere. The DF coordinates the tooth eruption by regulating osteoclastogenesis and osteogenesis [2]. After tooth eruption, DF cells come in contact with root dentin through perforated Hertwig's epithelial root sheath
(HERS) and then differentiate into cementum, periodontal ligament and part of the alveolar bone [3-8].

To date, the mechanisms of DF cells differentiated to periodontium remain undefined [7]. It is known that the interaction between HERS and the apical papilla provides the driving force for root elongation. The differentiation of DF cells always coordinates with root development [3]. Some studies 
have found that HERS could facilitate the cementogenic/osteogenic differentiation of periodontal ligament stem cells, which are the daughter cells of the dental follicle progenitor cells [5]. However, whether differentiation of DF cells could be regulated by the signals from the apical papilla during root elongation has been seldom investigated.

Dental follicle stem cells (DFSCs) are one kind of dental mesenchymal stem cell identified from DF tissues and have the capability to differentiate into osteoblast, periodontal ligament fibroblast, and cementoblast [1, 7-9]. Several signaling pathways, including BMP, Notch and canonical Wnt, are crucial for osteogenic differentiation of DFSCs [7]. WNT5a, a ligand that can activate both canonical and non-canonical Wnt pathways [10], is expressed in the dental epithelium and mesenchyme at early developmental stages of a tooth $[11,12]$. It is also one of the few Wnt molecules that are expressed in matured periodontium [13]. The capability of inducing mineralization of DFSCs shows WNT5a plays important roles in cytodifferentiation of DFSCs [13]. However, the expression patterns of WNT5a and related Wnt signaling genes in undifferentiated DF and developing periodontium tissues remain unclear. COL-I is the well-known differential marker of both osteogenesis and fibrogenesis [14-16]. Therefore, it is significant to study the expression pattern of COL-I during the root development as well.

Currently, most studies on tooth development are carried out on mice. However, the incisor's crown of mice grows continuously throughout life and is not the ideal model to study root and periodontium development. Recently, miniature pigs have been regarded as a new excellent experimental animal model in craniofacial research because they are similar to humans with respect to mandible anatomy and diphyodont dentition [17, 18]. Similar to humans, miniature pigs have more than one incisor in each quadrant, which are embedded deeply inside the alveolar bone. In this research, we use the third deciduous incisor (DI3) of miniature pig to elucidate the factors regulating the differentiation of DF cells during root development. First, the morphology of DF was examined, and then, the expression patterns of WNT5a, $\beta$-Catenin and COL-I in the undifferentiated DF cells and developing periodontium were determined and compared. To study the possible role of the apical papilla on DFSCs differentiation, the DFSCs were co-cultured with stem cells of the apical papilla (SCAPs), and the osteogenesis and fibrogenesis abilities of DFSCs were analyzed. Together, these results might provide insight into the complex interactions between the DF and the apical papilla during periodontium development.

\section{Materials and methods}

\section{Animals}

Pregnant and new born miniature pigs were obtained from the Animal Science Institute of Chinese Agriculture University. The gestation time was calculated starting from the insemination day. Pregnancy was proved by B-type ultrasonic inspection. Animal experiments were approved by the Animal Care Use Committee of Capital Medical University (Beijing, China) (Permit Number: AEEI-2016-063). The miniature pigs were anesthetized and sacrificed as previously described [19]. The third deciduous incisor (DI3) and dental tissues were isolated at embryonic day 90 (E90) and postnatal day 10 (PN10).

\section{Tissue preparation for histological analyses}

Samples were fixed in $4 \%$ paraformaldehydePBS at $4^{\circ} \mathrm{C}$ overnight. After being rinsed in PBS twice, the samples were decalcified with 10\% EDTA-PBS for 30-90 days according to the degree of calcification. They were then dehydrated through serial ethanol (30\%, 50\%, 75\%, 90\% and 100\%) and embedded in paraffin. The samples were sectioned ( $5 \mu \mathrm{m}$ thickness) and ready for staining. Sections were stained with hematoxylin and eosin (H\&E) for morphological examination.

\section{In situ hybridization}

The procedure for in situ hybridization was described previously [20]. Briefly, RT-PCR was performed using mRNA from tooth germ of miniature pigs. The correct size bands were extracted from agarose gels and DNA sequencing was performed. The RNA probe was synthesized by in vitro transcription according to the protocol of DIG RNA labeling Mix (Roche). For the staining procedure, after serial rehydration, the slides were treated with proteinase $\mathrm{K}(1 \mu \mathrm{g} / \mathrm{ml}$ in PBS) for $30 \mathrm{~min}$ at $37^{\circ} \mathrm{C}$. After being re-fixed with $4 \%$ paraformaldehyde, the sections were dehydrated in series of ethanol $(25,50,75$ and $100 \%)$. After being dried for $1 \mathrm{~h}$, the specimens were hybridized with probe at $70^{\circ} \mathrm{C}$ overnight. After being washed for hours, the sections were incubated with alkaline phosphatase conjugated anti-digoxigenin Fab (Roche) overnight. Signals were detected with NBT/BCIP substrates (Promega). Primers used for RT-PCR were list as follows:

- WNT5a (forward: 5'-ctggcaggactttctcaagg-3'; reverse: $5^{\prime}$-cgcgctgtcatacttctcct-3');

- $\beta$-Catenin (forward: 5'-ggtccatcagctttccaaaa-3'; reverse: $5^{\prime}$-ctgaacaagggtcccaagaa-3'); 
- Axin2 (forward: 5'-gagggagaaatgcgtggata-3'; reverse: $5^{\prime}$-tgggtgagagtttgcacttg-3').

\section{Immunohistochemistry}

The procedure for immunohistochemistry was described previously [19]. Briefly, sections were deparaffinized and treated with antigen retrieval, followed by immersed in $10 \% \mathrm{H}_{2} \mathrm{O}_{2}$ / methanol for 10 minutes to quench the endogenous peroxidase activity. The sections were incubated with primary antibodies at $4^{\circ} \mathrm{C}$ overnight. The primary antibodies were list as follows: WNT5a (LS-B4565, lifespan bioscience); $\beta$-Catenin (ab22656, abcam); COL-I (C2456, sigma).

\section{Cell culture}

The methods of isolation and stem cell characterization of DFSCs and SCAPs were described previously [21, 22]. Briefly, the DF tissue was harvested from the apical area of DI3, and apical papilla was obtained from apical part of dental papilla. After the tooth was extracted, the dental follicle tissue was attached to the root apex. We scraped the dental follicle tissue from the surface of root sheath and collected it. Then, we opened the root sheath carefully with forceps and dissected the apical papilla of $2 \mathrm{~mm}$ thickness. These tissues were digested with dispase II (4mg/ml, Sigma) and collagenase type I ( $3 \mathrm{mg} / \mathrm{ml}$, Sigma) for $1 \mathrm{~h}$ at $37^{\circ} \mathrm{C}$. Then the slurry was filtered through $70 \mu \mathrm{m}$ cell strainer (BD bioscience) and centrifuged at 1,100 rpm for $5 \mathrm{~min}$. Single cell suspensions were seeded into culture dishes and cultured with alpha-modification of the Eagle's medium (Invitrogen) containing 15\% fetal bovine serum, $2 \mathrm{mM}$ glutamine, $100 \mathrm{U} / \mathrm{mL}$ penicillin, and 100 $\mathrm{mg} / \mathrm{mL}$ streptomycin. The cells were incubated in 5\% carbon dioxide at $37^{\circ} \mathrm{C}$. The expression of stem cell markers and the capabilities of multi-lineage differentiation were determined.

\section{Co-culture and cell sorting}

To label SCAPs with green fluorescent protein, recombinant retrovirus expressing green fluorescent protein (RV-GFP, GenePharma) was used to label the third-passage SCAPs. Positive cell percentage was calculated above $95 \%$ by flow cell sorter. Then the third passage of SCAPs and DFSCs were seeded as the ratio of 1:1 in 6-well culture plate and co-cultured for 3 days. Then, the mixed cells were harvested and single-cell suspensions ( $1 \times 10^{6}$ cells) were incubated with anti-GFP tag antibody (mouse IgG2a) (66002-1-Ig, Proteintech). Subsequently, the mixed cells were washed and suspended in PBS containing $1 \%$ BSA $\left(80 \mu \mathrm{L} / 10^{7}\right.$ cells), before $20 \mu \mathrm{L}$ of anti-mouse IgG superparamagnetic MicroBeads (Miltenyi Biotec) per $10^{7}$ total cells was added. The sample was incubated for 15 minutes at $4^{\circ} \mathrm{C}$. Thus, the fraction of $\mathrm{GFP}^{+}$SCAPs, not DFSCs, was marked with superparamagnetic MicroBeads. Then, the mixed cell suspension was loaded onto a MACS ${ }^{\circledR}$ Column (Miltenyi Biotec), and placed in the magnetic field of a MACS Separator (Miltenyi Biotec). In the magnetic field, the flow-through contained the unlabeled DFSCs only. After removing the column from the magnetic field, the magnetically retained cells, which were $\mathrm{GFP}^{+}$SCAPs, were collected by pushing the plunger into the column. Thus, both of the fractions were collected separately. The percentage of $\mathrm{GFP}^{+}$ SCAPs was analyzed by fluorescence-activated cell sorting and the percentage enrichment was up to $98.14 \%$.

\section{Real-time RT-PCR}

Control DFSCs and co-cultured DFSCs were harvested and total RNA was extracted, followed by reverse transcription using the SuperScript III first-Strand synthesis system (Invitrogen). Real time RT-PCR was performed with SYBR GreenPCR mix (Applied Biosystems) and reactions were run on the CFX96real-time system (Bio-Rad). Triplicate reactions (20 $\mu \mathrm{l}$ volume) were performed. Melting curve analysis was completed. Expression level of each gene was normalized by the level of $\beta$-Actin. Relative expression level was determined using the $2^{\triangle \triangle \mathrm{CT}}$ method. Forward and reverse primers for WNT5a, $\beta$-Catenin, Alkaline phosphatase (ALP), Osteocalcin (OCN), Bone sialoprotein II (BSP-II), RUNX2, Periodontal ligament associated protein 1 (PLAP1)/asporin, FGF2, COL-I and $\beta$-Actin were list in Table S1.

\section{Statistical analysis}

Statistical analysis was performed using SPSS 13.0 software. Unpaired t-test was used for statistical significance. $P<0.05$ was considered statistically significant.

\section{Results}

\section{Morphological changes of the DF during root development}

The Development of tooth root begins following crown formation. We used the third deciduous incisor (DI3) of miniature pig as a model to examine the morphological changes of the DF during root and periodontium development. At the stage of E90, the crown calcification was almost completed. Enamel, dentin, ameloblasts and odontoblasts could be observed in the crown. The DF was located outside of the outer enamel epithelium (OEE) and surrounded the tooth germ as a sphere. The morphology was more obvious beneath the dental papilla (Fig.1A and 
$\mathrm{A}^{\prime}$ ). The DI3 erupted at E95 (the embryo was given birth at about E110). At the stage of postnatal day 10 (PN10), the morphology of the root apex region varied drastically. The DF was shaped like a crescent and was located between the apical foramen (AF) and the alveolar bone. At the lateral coronal corner of the $\mathrm{DF}$, the loose DF tissue was differentiating into compact periodontium. The perforated HERS and the cellular cementum could be observed on the surface of dentin (Fig.1B and $\mathrm{B}^{\prime}$ ).

\section{Expression dynamics showing high expression levels of WNT5a, $\beta$-Catenin, and COL-I in developing periodontium compared to the undifferentiated DF.}

To explore the factors influencing the differentiation of $\mathrm{DF}$, we studied the expression patterns of WNT5a, $\beta$-Catenin and COL-I and compared the differences between developing periodontium and the undifferentiated DF (DF beneath the $\mathrm{AF}$ ). We found that the expression of
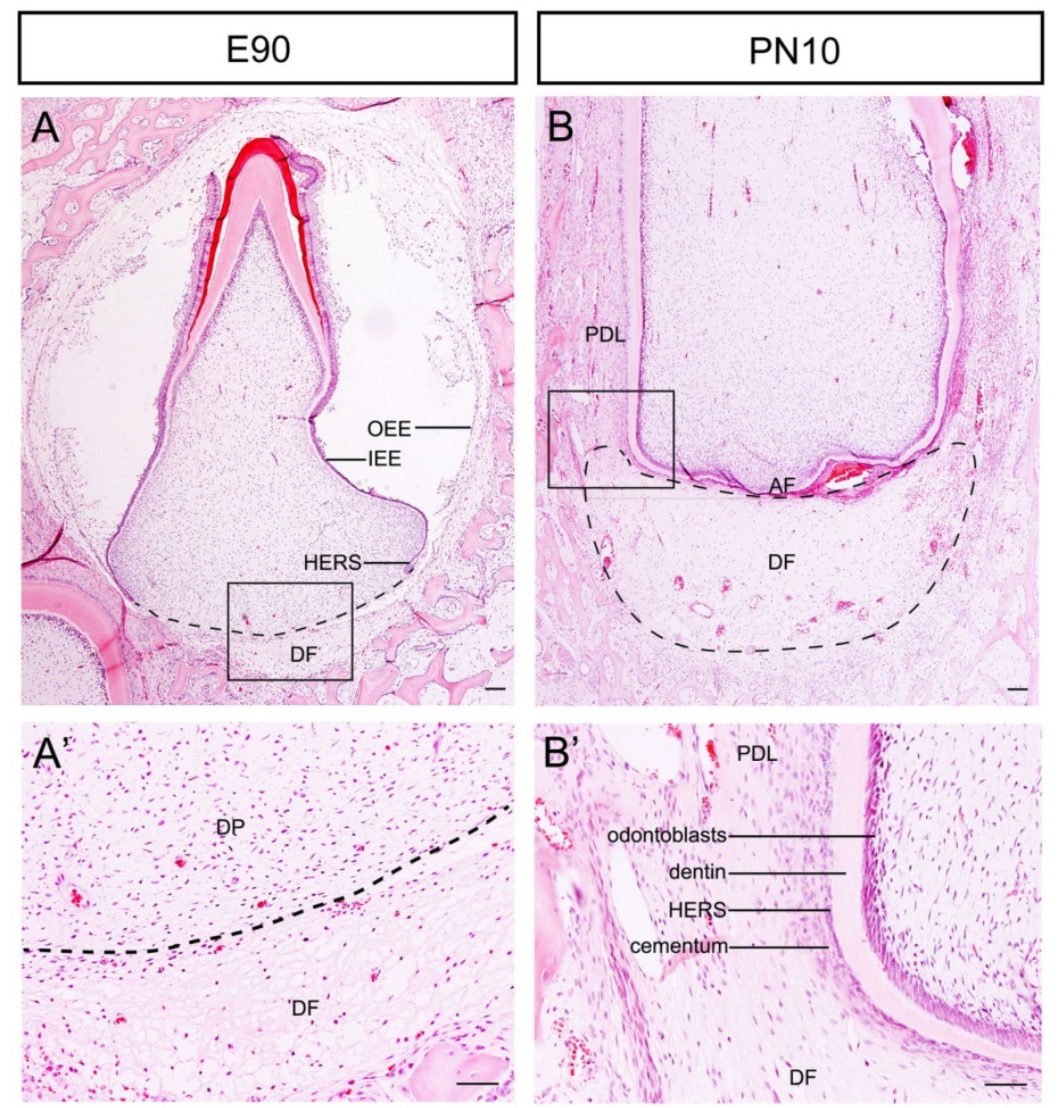

Figure 1. Morphological changes of the DF during root development of DI3 of miniature pig. (A, $A^{\prime}$ ) H\&E staining of DI3 at stage of E90; the boxed region in A was magnified in $A^{\prime}$. The dental follicle (DF) was located outside of outer enamel epithelium (OEE) and beneath the dental papilla (DP) (boxed regions). HERS grew apically and directed root formation. (B, B') H\&E staining of DI3 at the stage of PN10; the boxed region in B was magnified in B'. The DF was shaped like a crescent and was located between the apical foramen (AF) and the alveolar bone. The lateral coronal corner of DF (boxed region) was the area of differentiation from DF to periodontium. Periodontal ligament (PDL), cementum, broken HERS, dentin, and odontoblasts could be identified. Scale bars represent $100 \mu \mathrm{m}\left(\mathrm{A}, \mathrm{A}^{\prime}, \mathrm{B}, \mathrm{B}^{\prime}\right)$.
WNT5a was strong in the mature periodontal ment, weaker in the developing periodontium, weakest in the DF beneath the AF (Fig.2A-A"). riodontium was significantly stronger than it was in

$\beta$-Catenin is the central component of the canonical Wnt signaling pathway [23]. We found that the expression of $\beta$-Catenin was stronger in the developing periodontium than it was in the $\mathrm{DF}$ beneath the AF $(P<0.05)$ (Fig.2C-C', D). COL-I is the important differentiation marker of both osteogenesis and fibrogenesis $[14-16,24]$. We found that COL-I was expressed strongly in the developing periodontium, which was also stronger than it was in the DF beneath the AF $(P<0.05)$ (Fig. 2E-E"', F). It is worth mentioning intensity became weaker when getting closer to the AF (Fig. 2A", $\mathrm{C}^{\prime \prime}, \mathrm{E}^{\prime \prime}$ ). To further analyze the mRNA expression pattern of WNT5a and related canonical Wnt signaling genes $(\beta$-Catenin, Axin2), we conducted in situ hybridization. The result confirmed that the expression levels of these genes in developing periodontium were higher than those in the DF beneath the AF $(P<0.05)$ (Figure S1A-F).

Taken together, these results showed higher expression levels of WNT5a, $\beta$-Catenin, and COL-I in developing periodontium. The low-tohigh gradient from the central to lateral parts suggested the possible regulatory role of the apical papilla in DF differentiation through the AF.

\section{DFSCs showing weaker differential abilities after being co-cultured with SCAPs}

To study the possible regulatory role of the apical papilla in DF differentiation, we designed a co-cultured experiment (Fig.3A-C). DFSCs were isolated from DF tissue of DI3 at the stage of PN10 and cultured (Fig.3D). Meanwhile, SCAPs were isolated from the apical papilla and cultured (Fig.3E). After being infected with GFP-expressing retrovirus (Fig.3B, F, G), SCAPs were co-cultured with DFSCs at the ratio of 1:1. After 3 days of culture, the mixed cells were harvested.

Before cell sorting, the mixed cell suspension was incubated with anti-GFP tag antibody (mouse IgG2a) and then 
with anti-mouse IgG superparamagnetic MicroBeads sequentially. Only the GFP+ SCAPs could be labeled with the MicroBeads (Fig.3B). Then the mixed cells were loaded onto the column. In the magnetic field, cells that passed through were the unlabeled DFSCs, while the magnetically labeled $\mathrm{GFP}^{+} \mathrm{SCAPs}$ were retained within the column. After the magnetic field was removed, the retained GFP-SCAPs were eluted later (Fig.3C). After being sorted, the percentage enrichment of co-cultured DFSCs was up to 85.42 \pm

\section{$3.6 \%$ (Fig.3H-J).}

Then, the expression levels of WNT5a, $\beta$-Catenin, the osteogenic markers (ALP, OCN, BSP-II and RUNX2) and the fibrogenic markers (PLAP1, FGF2 and $(C L-I)$ were analyzed and compared between control and co-cultured DFSCs. The results showed that all of these markers were significantly down-regulated in the DFSCs co-cultured with SCAPs as compared to controls (Fig.4A-C).
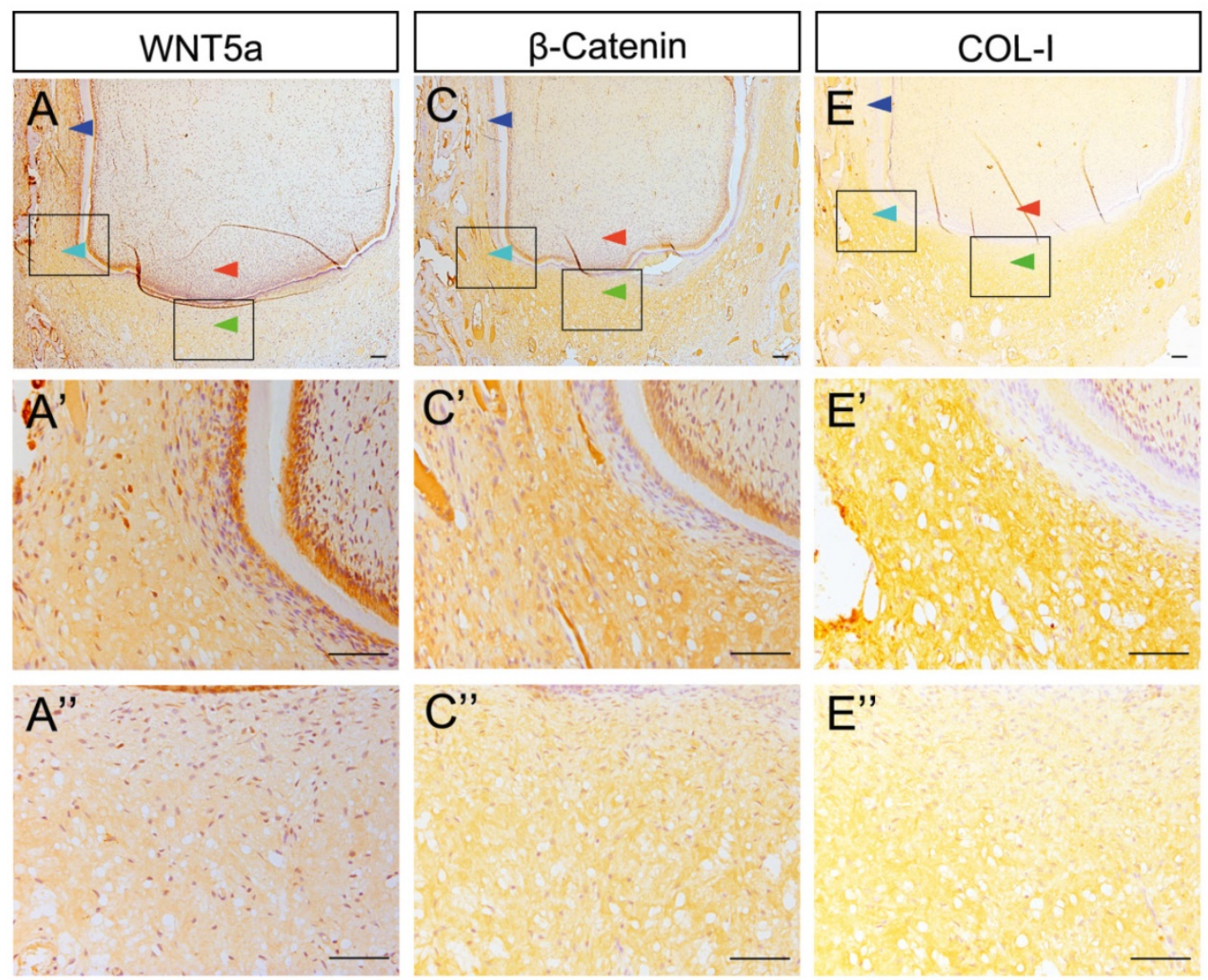

B

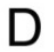

$\mathrm{F}$
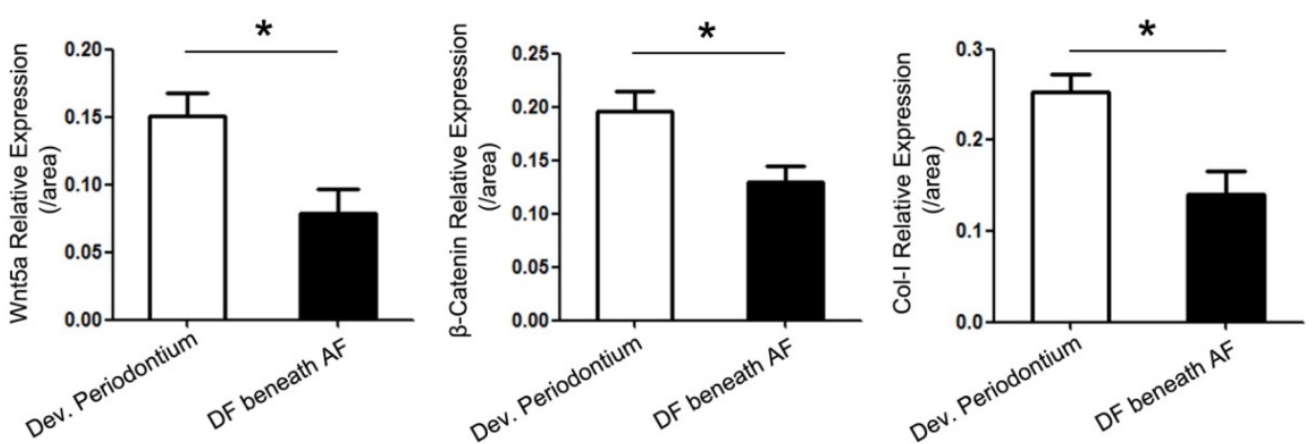

Figure 2. Expression dynamics of WNT5a, $\beta$-Catenin, and COL-I in developing periodontium and the undifferentiated DF. The differences of expression patterns of WNT5a, $\beta$-Catenin and COL-I between developing periodontium (Dev. Periodontium) and the DF beneath AF (undifferentiated DF) were studied. The boxed regions of Dev. Periodontium were magnified in A', C', E', and the boxed region of DF beneath AF was magnified in A', C', E'. Dark blue arrows point to the mature periodontium; light blue arrows point to the developing periodontium; green arrows point to the undifferentiated DF; red arrows point to the apical papilla. (A-A", B) WNT5a expression pattern showed the gradually increasing expression levels from the undifferentiated DF to the matured periodontal ligaments. The expression level in Dev. Periodontium was stronger than that in the DF beneath $A F(n=4, P<0.05)$. (C-C”, $D) \beta-C a t e n i n$ pattern showed the expression level in DF was higher than that in the periodontium. The expression level in Dev. Periodontium was stronger than that in the $D F$ beneath $A F(n=3, P<0.05)$. $(E-E "$, F) Col-I pattern showed the expression level in the DF was higher than that in the periodontium. The expression level in Dev. Periodontium was stronger than that in the DF beneath AF ( $n=3, P<0.05)$. Scale bars represent $100 \mu \mathrm{m}$ (A-A", C-C”, E-E”). 
A

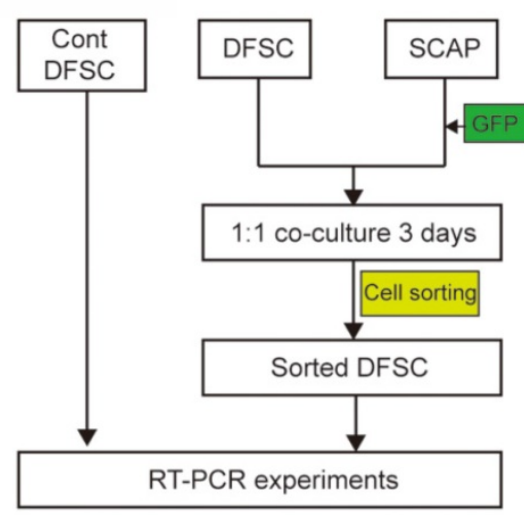

B

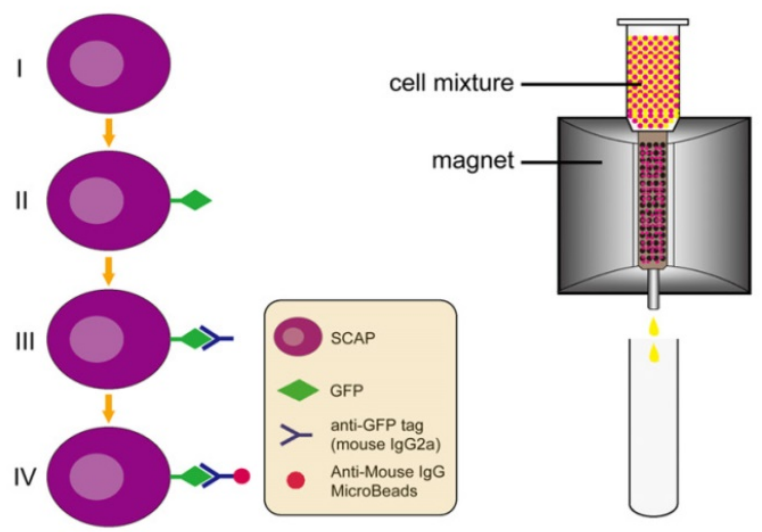

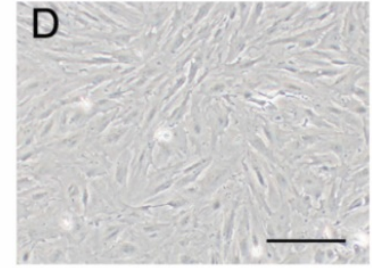
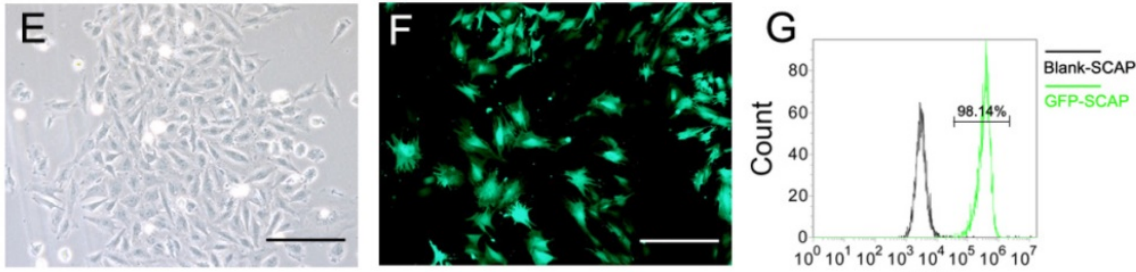

GFP
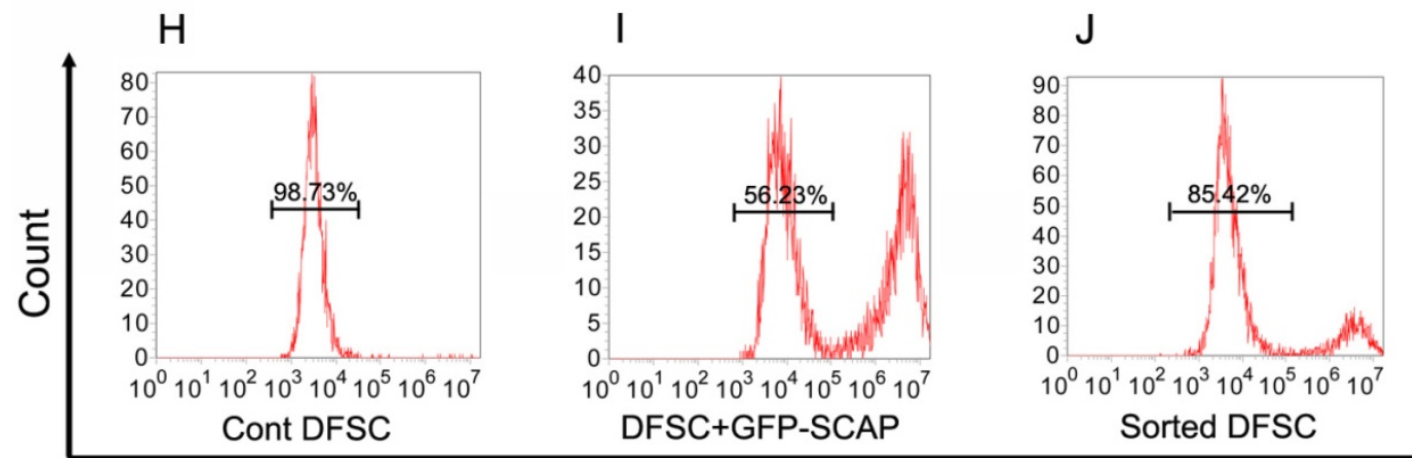

\section{GFP}

Figure 3. DFSCs were co-cultured with GFP-labeled SCAPs and then sorted. (A) The co-culture experiment was designed to study the possible regulating role of SCAPs in differentiation of DFSCs. (B-C) The schematic figures showing how to label SCAPs to sort them out from the cell mixture. (B) Step I-ll: SCAPs were transfected with recombinant retrovirus expressing GFP (RV-GFP). Step III: After the cell mixture (SCAPS:DFSCs $=1: 1$ ) was harvest, it was incubated with anti-GFP tag antibody (mouse lgG2a). Step IV: The mixture was incubated with anti-mouse lgG MicroBeads. (C) The cell mixture was loaded onto the column. The GFP+ SCAPs with MicroBeads (red) was attracted and retained inside the column in the magnetic field, while the unlabeled DFSCs (yellow) could pass through. After the magnetic field was removed, the retained SCAPs could be collected later. (D) DFSCs were isolated from DF tissue of DI3 at the stage of PNI0 and cultured. (E) SCAPs were isolated from the apical papilla of DI3 at the stage of PN10 and cultured. (F) SCAPs were transfected with GFP retrovirus. (G) High efficiency of GFP transfection $(98.14 \pm 3.3 \%$ ) was examined. ( $\mathrm{H}-\mathrm{J})$ After being sorted, the proportion of co-cultured DFSCs was elevated from $56.23 \pm 2.6 \%$ (before) to $85.42 \pm 3.6 \%$ (after). Scale bars represent $100 \mu \mathrm{m}$

\section{Discussion}

In the present study, the third deciduous incisor of miniature pig was used as the model to investigate the factors influencing DF differentiation during root development. We found that the expression levels of WNT5a, $\beta$-Catenin, and COL-I gradually increased from the undifferentiated DF to the developing periodontium. The osteogenesis and fibrogenesis abilities of DFSCs were inhibited when being co-cultured with SCAPs. It suggests that the apical papilla may help sustain the undifferentiated status of
DF before root development completes.

The DF is a kind of loose connective tissue sac that surrounds the tooth germ in the early developmental stages [1]. It coordinates tooth eruption by facilitating more bone resorption in the coronal half of the DF and more osteogenesis in the basal half $[2,25]$. A previous study found that dental follicle cells participated in tooth eruption via the Runx2-miR31-SATB2 loop [26]. After tooth eruption, cells in the DF differentiate into cementoblasts, fibroblasts and osteoblasts in the periodontium [3]. However, the morphologic dynamics of the DF 
during root development is largely unknown. With miniature pig as an animal model in this study, we found that the DF tissue was shaped like a crescent that was located between the root apical and the alveolar bone during root development (Fig.1 and Fig.5).

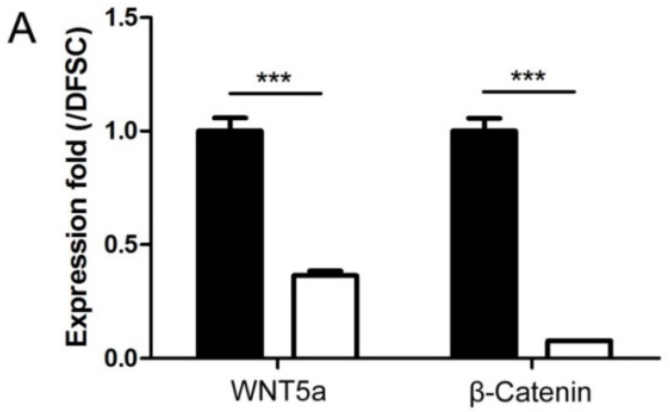

B
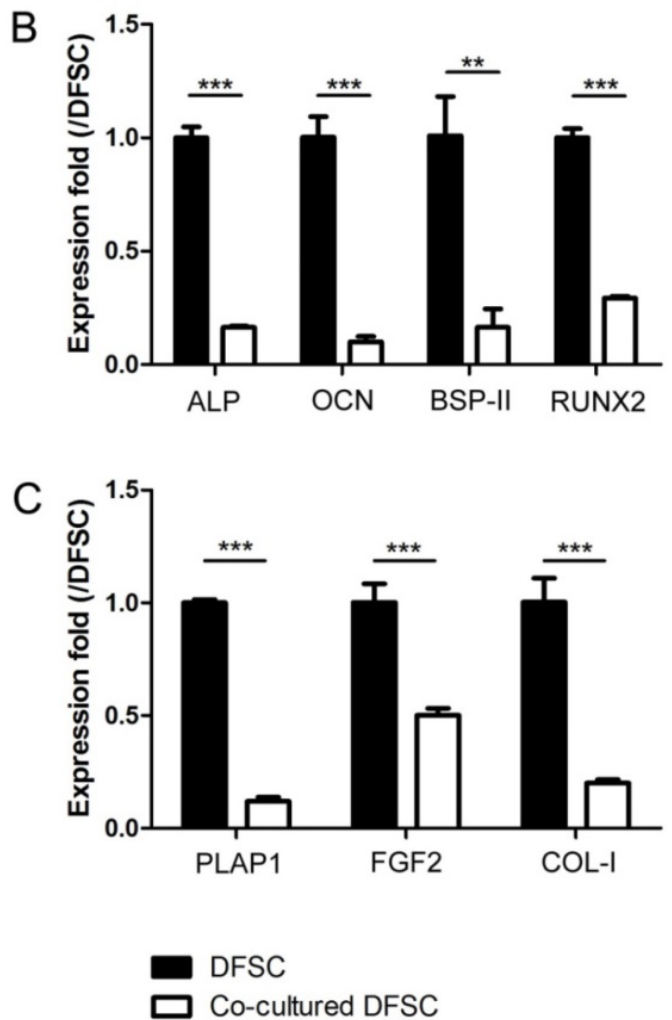

Figure 4. The expression levels of Wnt signaling and differentially related genes of DFSCs were down-regulated after being co-cultured with SCAPs. (A) The expression levels of WNT5a and $\beta$-Catenin of DFSCs were down-regulated after being co-cultured with SCAPs $(* * * P<0.001)$. (B) The expression levels of the osteogenic markers (ALP, OCN, BSP-II and RUNX2) of DFSCs were down-regulated after being co-cultured with SCAPs $(* * * P<0.001$, ** P<0.01). (C) The expression levels of the fibrogenic markers (PLAPI, FGF2 and COL-I) of DFSCs were down-regulated after being co-cultured with SCAPs $(* * * P<0.001)$

To date, the mechanisms of DF cells differentiated to periodontium remain largely unknown. In this study, we found that the lateral coronal corner of the DF was the central region where the differentiation into periodontium happens. The contact of DFSCs with broken HERS and dentin surface may facilitate the differentiation process. High expression levels of WNT5a, $\beta$-Catenin, and COL-I in this region showed these genes may play important roles in the proliferation and differentiation of DFSCs (Fig.5). In other studies, stimulation with WNT5a suppressed osteoblastic differentiation, while significantly enhancing the proliferation and the expression of PDL-related genes, such as Periostin, COL-I, and Fibrillin 1 [27]. In addition, it has been shown that canonical Wnt signaling could inhibit osteogenesis and cementoblast differentiation, while the inhibition of Wnt signaling by Dkk1 facilitates the cementogenic and osteogenic differentiation of DFSCs $[23,28]$. Due to the differentiation of multiple cell types in periodontium, the elaborate regulatory role of Wnt signaling in balancing osteogenesis and fibrogenesis needs to be further investigated.

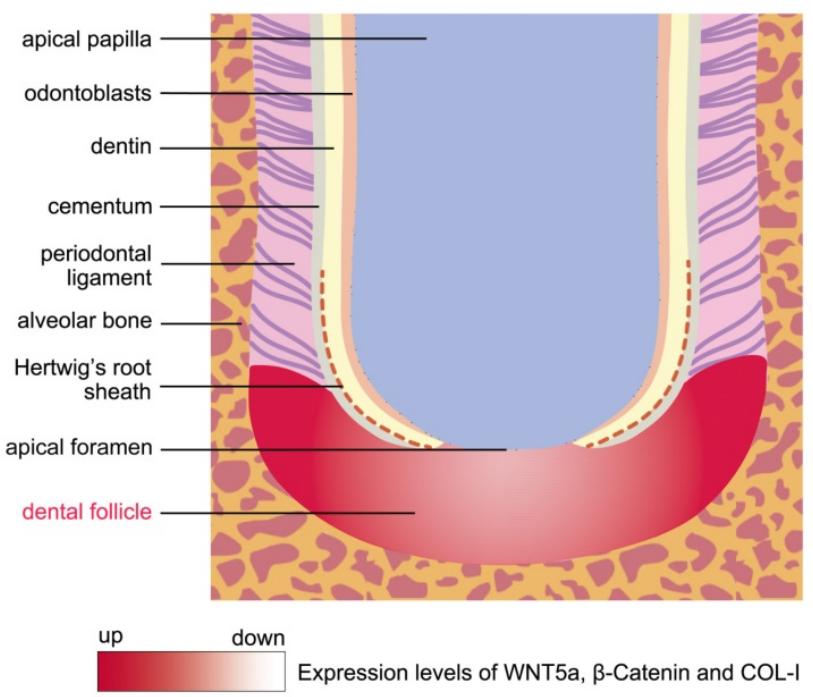

Figure 5. The schema of morphology and expression dynamics from the undifferentiated DF to the developing periodontium. The DF was shaped like a crescent and was located between the root apex and the alveolar bone during root development. The molecules of WNT5a, $\beta$-Catenin and COL-I were expressed strongly in the developing periodontium, which was located at the lateral coronal corner of the DF. Conversely, these genes were expressed at low levels in the region of DF beneath the AF.

How the DF coordinates with root apex to promote periodontium development, root elongation and bone remodeling remains poorly understood [3]. In this study, we found that the AF was the channel where the potential interaction between the apical papilla and the DF occurs. In the classical theory, the root apex formation and apical closure complete after root development. The size of the AF shrinks into a small hole as the root development completes [29]. This may suggest the potential communication between the inside and outside tissues of the AF during root development. To further investigate whether the differentiation abilities of DFSCs could be 
regulated by SCAPs, these cells were co-cultured and the differentiation abilities of DFSCs were examined. The differentiation abilities were evaluated by the expression levels of ALP, OCN, BSP-II and RUNX2, which are well known as osteogenic-specific genes [24, 30], and levels of PLAP1, FGF2 and COL-I, which are regarded as the fibrogenic markers. Among these genes, PLAP1 is known as the negative regulator of periodontal ligament mineralization and thus becomes one of the fibrogenic markers in the differentiation towards fibroblasts [31, 32]. The enhanced expression of FGF2 also corresponds to the differentiation of periodontal ligament fibroblasts [33]. In addition, COL-I is a well-known marker for fibroblast differentiation [16]. The RT-PCR results shows that the SCAPs may help sustain the undifferentiated state of DFSCs. Similarly, the pluripotency stem cell markers, including Oct4, Sox2 and Myc, were elevated in both dental papilla cells and DFSCs after being co-cultured [34]. This elevation confirmed our results that dental papilla cells helped maintain the undifferentiated status of DFSCs. However, the concrete interaction mechanisms between these two types of tissues/cells need to be further investigated in future studies.

Mice have one incisor in each quadrant and the crown grows continuously throughout life. The stem cells reside in the cervical loop and continue to differentiate into ameloblasts [35]. Different from mice, the growth of the incisor's crown in human and miniature pig is limited. The structure of the cervical loop is lost after crown formation completes and becomes HERS, which directs root formation. As a result, the incisors of miniature pig can be regarded as the ideal model to study the root and periodontium development of the anterior tooth in large mammals. Among three deciduous incisors, DI3 develops and erupts earliest [18]. Therefore, DI3 was selected as the study model in our research. In this study, we examined the morphology of the DF and the periodontium during DI3 root development and investigated the potential interaction between the DF and the apical papilla. These results may shed light on the future study of root and periodontium regeneration in large mammals.

\section{Abbreviations}

DFSCs: dental follicle stem cells; SCAPs: stem cells of apical papilla; DF: dental follicle; DI3: the third deciduous incisor; AF: apical foramen; DP: dental papilla; PDL: periodontal ligament; HERS: Hertwig's epithelial root sheath; IEE: inner enamel epithelium; OEE: outer enamel epithelium; H\&E: hematoxylin\& eosin.

\section{Supplementary Material}

Supplementary figure and table.

http://www.medsci.org/v15p0291s1.pdf

\section{Acknowledgments}

This work was supported by grants from National Natural Science Foundation of China (No. 81400478 and 91649124); Beijing Municipality Government grants (Beijing Scholar Program- PXM20 16_014226_000034, PXM2016_014226_000006, PXM 2015_014226_000116, PXM2015_014226_000055, PXM2 015_014226_000052, PXM2014_014226_000048, PXM20 14_014226_000013, PXM2014_014226_000053, Z12110 0005212004, PXM2013_014226_000055, PXM2013_ 014226_000021, PXM 2013_014226_07_000080 and TJSHG201310025005), Beijing Postdoctoral Research Foundation of China (2016 ZZ-46); Grants from Shanghai Key Laboratory of Stomatology (2015-kf01).

\section{Competing Interests}

The authors have declared that no competing interest exists.

\section{References}

1. Honda MJ, Imaizumi M, Tsuchiya S, et al. Dental follicle stem cells and tissue engineering. J Oral Sci. 2010; 52: 541-52.

2. Wise GE. Cellular and molecular basis of tooth eruption. Orthod Craniofac Res. 2009; 12: 67-73.

3. Li J, Parada C, Chai Y. Cellular and molecular mechanisms of tooth root development. Development. 2017; 144: 374-84.

4. Hammarstrom L, Alatli I, Fong CD. Origins of cementum. Oral Dis. 1996; 2: 63-9.

5. Sonoyama W, Seo BM, Yamaza T, et al. Human Hertwig's epithelial root sheath cells play crucial roles in cementum formation. J Dent Res. 2007; 86: 594-9.

6. Luan X, Ito Y, Diekwisch TG. Evolution and development of Hertwig's epithelial root sheath. Dev Dyn. 2006; 235: 1167-80

7. Morsczeck C. Molecular mechanisms in dental follicle precursor cells during the osteogenic differentiation. Histol Histopathol. 2015; 30: 1161-9.

8. Yao S, Pan F, Prpic V, et al. Differentiation of stem cells in the dental follicle. J Dent Res. 2008; 87: 767-71.

9. Morsczeck C, Gotz W, Schierholz J, et al. Isolation of precursor cells (PCs) from human dental follicle of wisdom teeth. Matrix Biol. 2005; 24: 155-65.

10. van Amerongen $\mathrm{R}$, Fuerer $\mathrm{C}$, Mizutani $\mathrm{M}$, et al. Wnt5a can both activate and repress Wnt/beta-catenin signaling during mouse embryonic development. Dev Biol. 2012; 369: 101-14.

11. Cai J, Mutoh N, Shin JO, et al. Wnt5a plays a crucial role in determining tooth size during murine tooth development. Cell Tissue Res. 2011; 345: 367-77.

12. Lin M, Li L, Liu C, et al. Wnt5a regulates growth, patterning, and odontoblast differentiation of developing mouse tooth. Dev Dyn. 2011; 240: 432-40.

13. Xiang L, Chen $\mathrm{M}, \mathrm{He} \mathrm{L}$, et al. Wnt5a regulates dental follicle stem/progenitor cells of the periodontium. Stem Cell Res Ther. 2014; 5: 135

14. Sun L, Zhang D, Liu F, et al. Low-dose paclitaxel ameliorates fibrosis in the remnant kidney model by down-regulating miR-192. J Pathol. 2011; 225: 364-77.

15. Long H, Sun B, Cheng L, et al. miR-139-5p Represses BMSC Osteogenesis via Targeting Wnt/beta-Catenin Signaling Pathway. DNA Cell Biol. 2017; 36: 715-24

16. Bhogal RK, Stoica CM, McGaha TL, et al. Molecular aspects of regulation of collagen gene expression in fibrosis. J Clin Immunol. 2005; 25: 592-603.

17. Wang S, Liu Y, Fang D, et al. The miniature pig: a useful large animal model for dental and orofacial research. Oral Dis. 2007; 13: 530-7.

18. Wang F, Xiao J, Cong W, et al. Morphology and chronology of diphyodont dentition in miniature pigs, Sus Scrofa. Oral Dis. 2014; 20: 367-79.

19. Wang F, Xiao J, Cong W, et al. Stage-specific differential gene expression profiling and functional network analysis during morphogenesis of diphyodont dentition in miniature pigs, Sus Scrofa. BMC genomics. 2014; 15: 103.

20. Wang F, Li Y, Wu X, et al. Transcriptome analysis of coding and long non-coding RNAs highlights the regulatory network of cascade initiation of permanent molars in miniature pigs. BMC genomics. 2017; 18: 148 
21. Tsuchiya S, Ohshima S, Yamakoshi Y, et al. Osteogenic differentiation capacity of porcine dental follicle progenitor cells. Connect Tissue Res. 2010; 51: 197-207.

22. Ding G, Liu $Y$, An $Y$, et al. Suppression of $T$ cell proliferation by root apical papilla stem cells in vitro. Cells Tissues Organs. 2010; 191: 357-64.

23. Silverio KG, Davidson $K C$, James RG, et al. Wnt/beta-catenin pathway regulates bone morphogenetic protein (BMP2)-mediated differentiation of dental follicle cells. J Periodontal Res. 2012; 47: 309-19.

24. Guillot PV, De Bari C, Dell'Accio F, et al. Comparative osteogenic transcription profiling of various fetal and adult mesenchymal stem cell sources. Differentiation. 2008; 76: 946-57.

25. Wise GE, Yao S. Regional differences of expression of bone morphogenetic protein-2 and RANKL in the rat dental follicle. Eur J Oral Sci. 2006; 114: 512-6.

26. Ge J, Guo S, Fu Y, et al. Dental Follicle Cells Participate in Tooth Eruption via the RUNX2-MiR-31-SATB2 Loop. J Dent Res. 2015; 94: 936-44.

27. Hasegawa D, Wada N, Maeda H, et al. Wnt5a Induces Collagen Production by Human Periodontal Ligament Cells Through TGFbeta1-Mediated Upregulation of Periostin Expression. J Cell Physiol. 2015; 230: 2647-60.

28. Nemoto E, Koshikawa Y, Kanaya S, et al. Wnt signaling inhibits cementoblast differentiation and promotes proliferation. Bone. 2009; 44: 805-12.

29. Trope M. Treatment of the immature tooth with a non-vital pulp and apical periodontitis. Dent Clin North Am. 2010; 54: 313-24.

30. Yin X, Wang X, Hu X, et al. ERbeta induces the differentiation of cultured osteoblasts by both Wnt/beta-catenin signaling pathway and estrogen signaling pathways. Exp Cell Res. 2015; 335: 107-14.

31. Yamada S, Tomoeda M, Ozawa Y, et al. PLAP-1/asporin, a novel negative regulator of periodontal ligament mineralization. J Biol Chem. 2007; 282: 23070-80.

32. Ueda M, Goto $\mathrm{T}$, Kuroishi $\mathrm{KN}$, et al. Asporin in compressed periodontal ligament cells inhibits bone formation. Arch Oral Biol. 2016; 62: 86-92.

33. Sowmya S, Chennazhi KP, Arzate H, et al. Periodontal Specific Differentiation of Dental Follicle Stem Cells into Osteoblast, Fibroblast, and Cementoblast. Tissue Eng Part C Methods. 2015; 21: 1044-58.

34. Peng Z, Liu L, Wei X, et al. Expression of Oct-4, SOX-2, and MYC in dental papilla cells and dental follicle cells during in-vivo tooth development and in-vitro co-culture. Eur J Oral Sci. 2014; 122: 251-8.

35. Li J, Feng J, Liu Y, et al. BMP-SHH signaling network controls epithelial stem cell fate via regulation of its niche in the developing tooth. Dev Cell. 2015; 33: 125-35. 